\title{
A new tool for variable multiple endmember spectral mixture analysis (VMESMA)
}

\author{
F. J. GARCÍA-HARO*†, S. SOMMER + and T. KEMPER \\ $\dagger$ Departament de Termodinàmica, Facultat de Física, Dr. Moliner, 50, \\ 46100 - Burjassot, Valencia, Spain \\ ‡EC Joint Research Centre, Institute for Environment and Sustainability, Soil \& Waste \\ Unit, TP 262 I-21020 Ispra (VA), Italy
}

(Received 7 October 2003; in final form 21 October 2004)

\begin{abstract}
Spectral mixture analysis is a widely used method to determine the sub-pixel abundance of vegetation, soils and other spectrally distinct materials that fundamentally contribute to the spectral signal of mixed pixels. In this paper we present a computing and environmental analysis tool, named VMESMA, which extends the possibilities of conventional spectral unmixing. The basis is the categorization of the scene into different units or scene sub-areas and software guidance for endmember selection, allowing for a better adaptation of the model to the conditions of the main cover types. For each pixel an individual combination of endmembers may be selected by automated matching to model quality criteria. This hierarchical assessment can incorporate a priori knowledge from different data sources, including information derived from the unmixing results. Based on an iterative feedback process, the unmixing performance may be improved at each stage until an optimum level is reached. VMESMA allows an immediate estimate of the proportions, which is very robust against external factors (e.g. illumination) and canopy shade. An application of VMESMA on hyperspectral data has been conducted to evaluate the possibilities to map residual sludge and sludge derivatives for two consecutive years with changing land surface conditions. The method offered greater flexibility and new possibilities to improve the understanding and modelling of the scene characteristics.
\end{abstract}

\section{Introduction}

The estimation of sub-pixel proportions of land surface elements in general, and of vegetation components in particular, has been largely addressed in the remote sensing literature. Spectral mixture analysis (SMA) has been developed in recent years to model the spectral variability in multi- or hyperspectral images, and to relate the results to the physical abundance of surface constituents represented by a small number of spectral endmembers (EMs), which correspond to the spectral characteristics of the scene cover types (Adams et al. 1986, Smith et al. 1990, Thomson and Salisbury 1993, Roberts et al. 1998). Unlike other techniques, SMA is especially adequate for dealing with scenes in which the spatial variability within a pixel is high. Thus it provides a means to detect and represent components that occur entirely at a subpixel level, such as sparse vegetation in an arid environment.

\footnotetext{
*Corresponding author. E-mail: J.Garcia.Haro@uv.es
} 
EM fractions can be used for classification and/or monitoring surface changes over time (Adams et al. 1995, DeFries et al. 2000). Finally, when supported with spectral ground truth data, SMA allows the repeatable and accurate extraction of quantitative subpixel information that can be incorporated into physical models of surface processes, which is the ultimate goal for most remote sensing data analysis.

Linear mixture is the basis hypothesis of some simple BRDF models (e.g. geometrical-optical (GO) or kernel-driven models). However, the degree of validity of this assumption depends primarily on (1) the presence of multiple scattering, (2) directional effects that can be expressed by variations of downwelling and upwelling radiative fluxes as a function of the solar and viewing directions, and (3) the scale size (Qin and Gerstl 2000).

In practice, the strategy to reflect the variety of optical properties of the most relevant scene elements in the EM selection is one of the key issues in the successful application of SMA, since the attainable accuracy is highly influenced by the spectral similarity between EMs. Different SMA approaches have been described in the literature. The most widely used method consists of employing a single set of EMs (typically between two and five) on the whole image, and using all available EMs at the same time. Though it is mathematically feasible to use in the decomposition as many EMs as spectral bands available, even with a small number of EMs noisy and highly correlated data exert a significant influence on the proportion estimates of the lowest contrast materials (Smith et al. 1994, GarcíaHaro et al. 1996, Drake et al. 1999). However, many vegetation types, soils and other components are present in the scene, in such a way that 2-5 EMs are often insufficient to describe the scene fully, and lead to unclear results. For example, fractions of the unmodelled EMs will be partitioned or allocated to incorrect EMs. Fractional errors result also in physically unrealistic negative or overpositive fractions. Simple unmixing is, in addition, unable to address correctly the natural variation of components in the scene. Smith et al. (1994) found that the dominant effect of EM spectral variability is expressed in changes in fractions rather than an increase of the modelling error.

In recent years, many authors have proposed a more complex model where both the number and the set of EMs vary on a per-pixel basis. Roberts et al. (1998) refer to this technique as multiple endmember spectral mixture analysis (MESMA). Initially the method was proposed to map several different types of green vegetation, non-photosynthetic vegetation, shade and soil EMs in semi-arid environments (Roberts et al. 1991, 1993). The idea consists of restricting the large set of possible EMs within a scene to a number of small sets of appropriate EMs that can be different for each pixel, thereby allowing an accurate decomposition using a virtually unlimited number of EMs. The dynamic identification of the optimum EM subset for each image pixel provides a more accurate interpretation of the mixed pixels than SMA, improving the fit of the model. Also the accuracy of fractions increases due to the increase of the probability that the exact number of EMs required to account for the sub-pixel spectral variability is utilized (Sabol et al. 1992, García-Haro 1997). Because the optimum subset for the spectral description of each pixel is always maintained, the number of components in the scene is only limited by the computational cost and the spectral separability between intra-class materials.

Different criteria for selecting the EMs in multiple EM submodels have been proposed by many authors in different studies (García-Haro et al. 1995, Hill et al. 
1995, Maselli 1998, Painter et al. 1998, Roberts et al. 1998). However, there are a number of methodological and practical application problems that often impede the operational use of MESMA:

1. While SMA is computationally modest, MESMA can be very CPU-intensive because it often implies the evaluation of tens of models for every image pixel (Okin et al. 1999). This may result in a large number of abundance images of different EMs, which increases the complexity of the analysis and may produce an undesired over-stratification of the scene.

2. Estimated local EMs may be incorrect. In general, authors consider, as a unique condition, that EMs characterize the overall variance in the image to the noise level. However, this is not sufficient since low modelling errors may coexist with relatively high fractional errors, e.g. when the spectrum of interest resembles a mixture of other materials, such as that of dry grass, which resembles mixtures of shade, green foliage, and soil (Ustin et al. 1993). Thus, one additional prerequisite is that EMs should reflect the different surface conditions in the scene.

3. As a consequence of the large variety of different objects or subunits that may be found in a scene, many EM contributions are needed to describe all possible objects. This slows down the process and also introduces some errors in the modelling of the pixels that do not contain these objects. EM candidates chosen to represent the spectral variability of one area of interest can overdetermine the spectral signal of most other scene areas (e.g. a higher number of EMs used than really represented in the pixel). An optimum strategy seems to be identifying different sub-areas and decomposing them in a separate stage. For example, for agricultural studies one possible procedure would consist of first classifying the pure pixels and then unmixing the mixed pixels (Klein-Gebbinck 1998).

Against this background a Variable Multiple Endmember Spectral Mixture Analysis (VMESMA) strategy for improved performance of the unmixing processes has been developed and implemented in a flexible data processing tool. VMESMA is based on a stratification of the area and a zone-dependent choice of multiple candidate submodels and unmixing algorithms, each valid within a scene sub-area. By formalizing some knowledge of the application domain into a simple scene model, the spatial relationships between the pixels can be used to meet the user requirements. In the following section we describe the concept of VMESMA in detail. An example of the application of VMESMA on hyperspectral data in the context of determining levels of heavy metal contamination after a mining accident is provided in $\S 3$. Finally, the conclusions are presented in $\S 4$.

\section{The VMESMA package - methodological concept and application tool}

The immanent problems of the overall spectral unmixing concept imply the necessity of a variable but nonetheless streamlined modelling strategy, in terms of optimized selection of EM sets taking into account the spectral and spatial variability of the image scenes. In the VMESMA concept in principle this includes:

- new ways of reducing the influence of external factors such as shading, brightness differences due to variability of surface roughness and terrain illumination by standardizing the spectra in the unmixing; 
Table 1. Summary of some relevant VMESMA features.

\begin{tabular}{ll}
\hline Use of spatial information & $\begin{array}{l}\text { Stratification driven by the targeted application } \\
\text { EM submodels adapted to physiographic conditions } \\
\text { Reduced EM misidentification }\end{array}$ \\
& $\begin{array}{l}\text { Reduced computation time } \\
\text { Addressing unmodelled EMs }\end{array}$ \\
Link with a thematic spectral library & $\begin{array}{l}\text { More reliable modelling of scene characteristics } \\
\text { Problem diagnosis and } \\
\text { solution }\end{array}$ \\
Hierarchical assessment & $\begin{array}{l}\text { Incorporation of prior knowledge and previous unmixing } \\
\text { results }\end{array}$ \\
& $\begin{array}{l}\text { Focussed analysis of specific subareas } \\
\text { Flexibility to improve model parameters }\end{array}$ \\
Advanced unmixing & Standardized unmixing \\
methodology & Use of contextual information \\
& Speed optimization capabilities
\end{tabular}

- robust, novel criteria to assess the performance quality of alternative EM sets in the selection process of optimum EMs for individual pixels; and

- guided and streamlined injection of a priori knowledge in the unmixing process.

Table 1 shows some relevant features of VMESMA, which will be described in detail here.

\subsection{The VMESMA scheme}

VMESMA is conceived as an iterative feedback process, in which unmixing performance may be potentially improved in each stage until an optimum level is reached. This permits the definition of standard and repeatable pathways to incorporate information dynamically derived from the most recent unmixing outcomes with other sources of data, in order to optimize the algorithms and increase the flexibility of the modelling approach. The structure of VMESMA is summarized in figure 1.

After each unmixing iteration, an abundance of similar EMs is usually merged to thematically group EMs into general categories, as it provides the physical context necessary to interpret the results. A suite of outputs is readily available to conduct the error assessment, mainly to evaluate the model performance and fractional errors. With this aim, the 'Linking tools' function provides overlay capabilities that show all the specified spectral information of the chosen pixel. Thus the researcher can seek for pixels with high rms or fractional errors, relating such results with simultaneous analysis of their reflectance spectra, best-fit modelled spectra, residual spectra, model chosen, unit number and set of abundances obtained.

Problematic areas are usually found from high modelling and abundance errors (typically identified from negative values or not matching the proportions derived from the field observations or other sources). This information can be used to incorporate new areas to the image segmentation. Next, new EMs can be extracted from these areas, which will be incorporated in the unmixing library. The subsequent iteration will be performed on the basis of the updated segmentation and using a modified models list extracted from the updated library. VMESMA enables selective unmixing in which only a few image subunits are processed, while leaving unaltered the abundance of the rest, as obtained in a previous iteration. This strategy may considerably speed up the computations. 


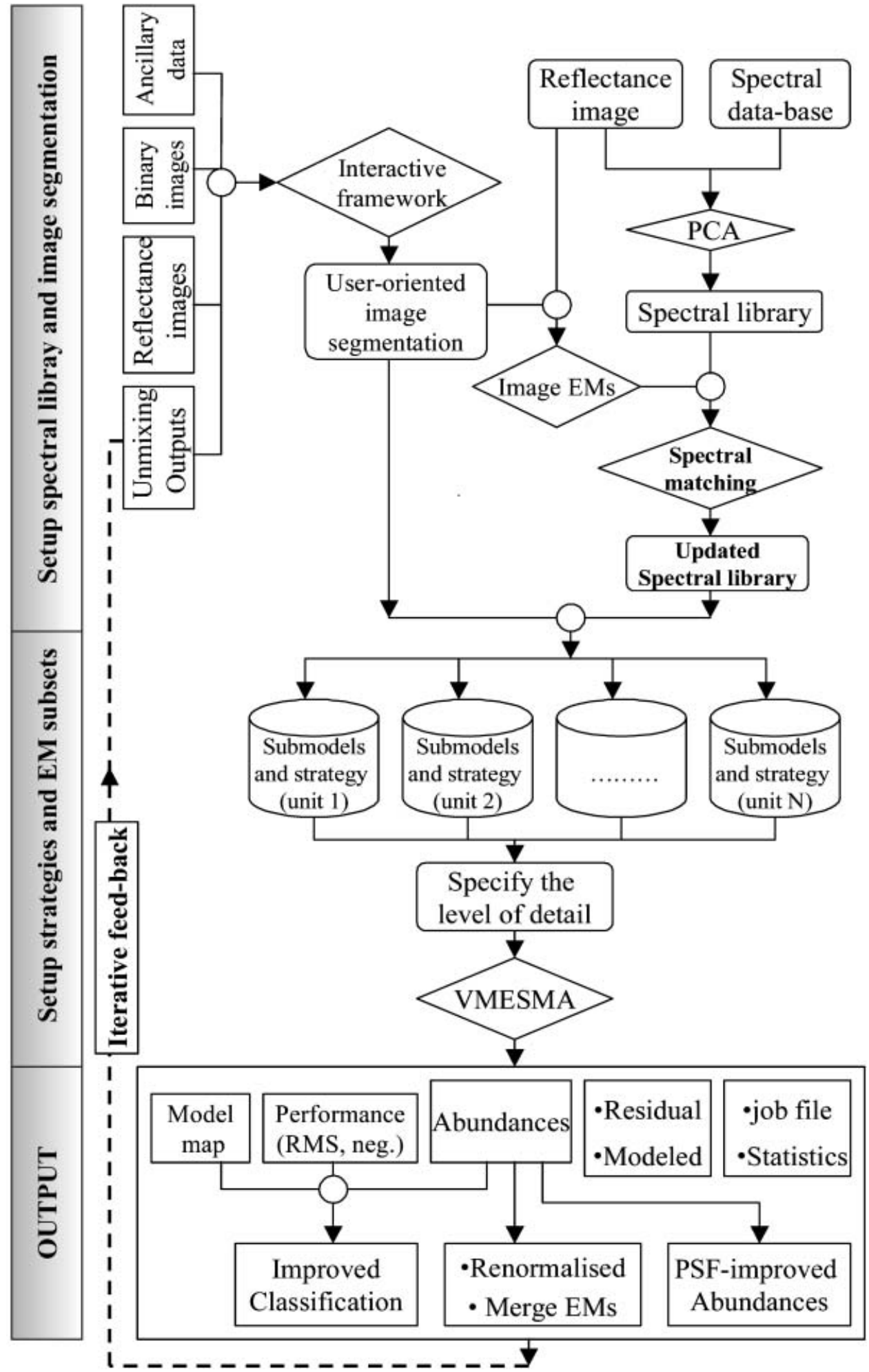

Figure 1. Flowchart of the working VMESMA scheme. 
The Unmixing frame (see figure 2) is an interface for specifying the EM submodels and the strategy to unmix each individual unit. Each unmixing is executed in a sequence unit by unit. For each individual unit the following parameters can be specified:

- The expert system used to select the best EM subset (see §2.4). Although VMESMA is based on an adaptable selection of the number of EMs (2, 3 or 4), a 'hard' criterion (e.g. selecting always a 3-EM model) or, alternatively, a simpler mixed criterion (e.g. enabling only 2 or 3 EM solutions) is also possible.

- The lists of candidate EM subsets (see \$2.6).

- The type of unmixing algorithm, i.e. conventional or standardized unmixing (see $\$ 2.2)$.

- The final criterion used to evaluate the model performance, i.e. rms residual, maximum residual or colinearity factor (see §2.3).

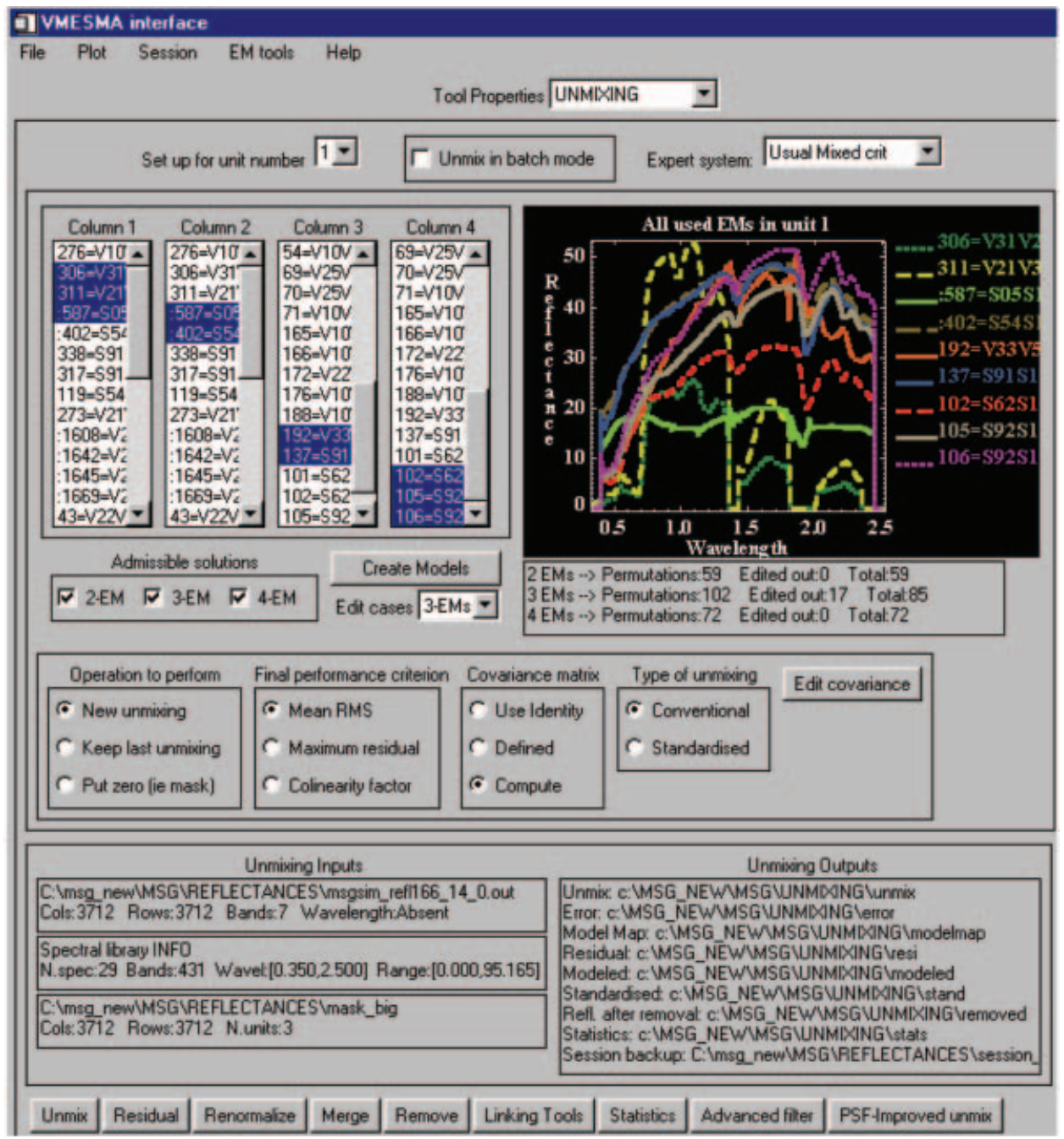

Figure 2. The Unmixing sheet allows specification of the EM submodels and a strategy to unmix each individual unit. The frame also provides helpful information on the unmixing parameters and specialized tools to unmix, visualize the results and improve them. 
- The task performed on each individual unit: (i) mask, (ii) unmix using the updated submodels list, or (iii) keep the abundances of the last unmixing (i.e. selective unmixing).

- The covariance matrix of the observations, e.g. to modify the relative contribution of each specific spectral band in the unmixing analysis (see \$2.7).

In summary, the segmentation of the scene in combination with a knowledgebased expert system adds flexibility to the solution. Hence the determination of the optimum pixel submodel is not a 'black-box' but it may be effectively assisted by unmixing outputs, field information, image characteristics and the targeted application.

\subsection{Conventional vs standardized unmixing}

VMESMA allows the application of conventional unmixing to estimate the EM proportions, based on reflectance, but it also provides an alternative solution, namely standardized unmixing, which offers new, more appropriate, solutions to specific problems. We now express briefly the formulation of conventional unmixing using matrix-vector notation, and then introduce the standardized unmixing.

2.2.1 SMA theory and equations. We will refer to $n$ as the dimensionality of the spectral observations and the column vector $r\left(r_{1}, r_{2}, \ldots, r_{n}\right)$ will be the measured spectrum of the mixture. Assuming that there are $c$ composing EMs within the mixture we will order their spectral response in an $n \times c$ matrix, $\boldsymbol{E}$, with the reflectance of the $c$ EMs in columns. Let $\boldsymbol{f}\left(f_{1}, f_{2}, \ldots, f_{c}\right)$ be the column vector with $c$ unknown proportions of the EMs in the mixture. The SMA assumes a linear dependence of the theoretical prediction $\boldsymbol{r}$ on the unknown parameter $f$ :

$$
\boldsymbol{r}=\boldsymbol{E} \boldsymbol{f}+\boldsymbol{\varepsilon}
$$

where $\varepsilon$ is the residual vector, which would ideally be a multivariate normal distribution with a mean of zero. This mixing equation is usually accompanied by two constraints. The normalization constraint says that a pixel is well defined by its components, whose proportions or abundances should therefore add up to unity:

$$
\sum_{i=1}^{c} f_{i}=1
$$

The positivity constraint says that no component of a mixed pixel can make a negative contribution:

$$
f_{i} \geq 0 \quad i=1, \ldots, c
$$

Let $\boldsymbol{V}(\boldsymbol{r})$ denote the error matrix of the observations $\boldsymbol{r}$, namely its covariance matrix. The least-square principle establishes that the unknown parameters are those that minimize the Mahalanobis distance between the pixel $\boldsymbol{r}$ and point $\boldsymbol{E} \boldsymbol{f}$ :

$$
\chi^{2}=(\boldsymbol{r}-\boldsymbol{E} \boldsymbol{f})^{\mathrm{T}} \boldsymbol{V}^{-1}(\boldsymbol{r}-\boldsymbol{E} \boldsymbol{f})
$$

A large number of solution methods exist for this type of linear problem, including least-squares estimation, singular value decomposition, Gram-Schmidt orthogonalization and factor analysis (Boardman 1989, Smith et al. 1990). However, not all solutions are independent. For example, the singular value decomposition is 
mathematically equivalent to the least-squares solution without constraints. In general, solutions that do not constrain the abundances to the normalization condition produce biased and highly noise-dependent solutions for the abundances (García-Haro 1997). VMESMA uses a partially constrained least-squares solution, which introduces in equation(4) a Lagrange multiplier to impose the sum-to-one constraint (García-Haro et al. 1996). This solution provides a unique and unbiased solution that is computationally fast due to its matricial form. However, abundances are not constrained to lie between 0 and 1. Thus, noise in the observations and inaccuracies of the model can bring about undesirable outcomes. We have preserved negative fractions since they may also convey useful information and provide an additional means of assessing model accuracy. Although numerical methods are available (Boardman 1989, Klein-Gebbinck 1998), the computational complexity of these methods often cannot be afforded with satellite data and moreover they introduce a bias in the solution (García-Haro 1997).

2.2.2 Problems derived from a shade EM. Shade refers to shadow cast by objects at all spatial scales as well as shading (variation in lightness) by topography, and the effects of soil/rock roughness. Hence, a spectral EM is usually required in the SMA to isolate the influence of shading and shadows. In the literature, its spectral characteristics are often approximated with a continuous spectrum of zero reflectance. Other authors have proposed a 10\% reflectance shade EM (Accioly et al. 1998). However, the shade 'contains' radiance scattered on soil by atmospheric components and canopy elements. Thus, reflectance of shaded soil is directly related to leaf transmittance and inversely proportional to density and opacity of leaves in the canopy, and can also increase with the turbidity of the atmosphere (García-Haro et al. 1999). A constant shade EM is unable to capture the spectral variability of shading and shadows, resulting in fractional errors since surfaces shadowed by plants are enriched in near-infrared radiations (Leblon et al. 1996).

Although shade conveys useful information regarding vegetation structure, a key to vegetation type identification and classification (Ustin et al. 1993, Adams et al. 1995, Shimabukuro et al. 1998, Roberts et al. 1998, Peddle et al. 1999), the use of a shade EM introduces important drawbacks:

1. It is difficult to define unambiguously an average pixel signature for the shade component, since the 'obscurity' or tonality of shadow cast by vegetation varies depending on the canopy structure and the leaves' transmittance (García-Haro and Sommer 2002).

2. The shade abundance is less directly interpretable in ecological terms than soil and vegetation maps. Moreover, shade is dependent on sun/view angles and topography, and varies with time.

3. Using a shade EM results in a more unstable solution of the SMA due to lowering of spectral complexity between EMs, since the shade EM, by definition a very dark spectrum, makes the mixing library less well conditioned (Boardman and Goetz 1991).

4. Retrieving the absolute abundances of the EMs requires partitioning of the shade abundance between the remaining components. One common procedure consists of re-scaling the remaining fractions so that they sum to one (i.e. multiplying by a renormalization factor $\left.F=1 /\left(1-f_{\text {shade }}\right)\right)$. For example, Camacho-de Coca et al. (2003) proposed a different renormalization factor that depends on the canopy structure. However, renormalizing the shade 
introduces systematic errors, since at the subpixel scale, where shadows cannot be spatially resolved, it is not possible to tell whether shade is due to shading, shadows, or to a combination of both (Smith et al. 1990).

2.2.3 An alternative procedure: Standardized SMA. We propose an alternative approach, which consists of performing a standardization on both the EMs and the image spectra as a previous step before applying the SMA. This standardization transforms the data to a set of variations about the mean value with a mean value of zero and a standard deviation of one. This allows matching of the data in a manner independent of the reflectance scale (Mackin et al. 1991):

$$
\hat{\boldsymbol{r}}=\frac{\boldsymbol{r}-\mu_{\mathrm{r}}}{\sigma_{\mathrm{r}}}
$$

where $\hat{\boldsymbol{r}}$ is the standardized pixel vector associated $\boldsymbol{r}$, with mean $\mu_{\mathrm{r}}$ and standard deviation $\sigma_{\mathrm{r}}$. We will refer to this new approach as standardized unmixing. This procedure enhances the information due to the spectral shape, such as gradient (i.e. derivative features) and absorption bands. Using the standardized EMs, $\widehat{\boldsymbol{E}}_{i}(i=1, \ldots, c)$, the unmixing is formulated:

$$
\widehat{\boldsymbol{r}}=\sum_{i=1}^{c} \widehat{\boldsymbol{E}}_{i} \widehat{f}_{i}+\widehat{\boldsymbol{\varepsilon}}
$$

where $\hat{f}_{i}$ is the proportion of such EM in the standardized coordinates, and $\widehat{\varepsilon}$ is the residual vector expressed in standardized units. The outputs are the proportions $\hat{\boldsymbol{f}}$ and the residuals $\hat{\mathcal{E}}$. Although the solution equations are similar to the conventional SMA, the normalization constraint is now expressed differently:

$$
\sum_{i=1}^{c} \frac{\hat{f}_{i}}{\sigma_{E_{i}}}=\frac{1}{\sigma_{\mathrm{r}}}
$$

By combining equations (5) and (6) we have:

$$
\frac{\boldsymbol{r}-\mu_{\mathrm{r}}}{\sigma_{\mathrm{r}}}=\sum_{i=1}^{c} \frac{\boldsymbol{E}_{i}-\mu_{\boldsymbol{E}_{i}}}{\sigma_{\boldsymbol{E}_{i}}} \widehat{f}_{i}+\widehat{\varepsilon}
$$

Thus the linear assumption implies that a simple relationship exists between $\boldsymbol{f}$ and $\hat{\boldsymbol{f}}$ :

$$
f_{i}=\frac{\sigma_{\mathrm{r}}}{\sigma_{E i}} \hat{f}_{i}
$$

As opposed to conventional SMA, standardized unmixing already accounts for differences in shadow or shade, thereby reducing the error of estimated abundances caused by the use of a shade EM. Since conventional and standardized unmixing convey different information, both strategies may be used to model mixed pixels, each one assessing opposite features. While conventional SMA is rather insensitive to spectral absorptions of minor constituents because the analysis predicts the bestfit least-squares regression over the full spectrum, standardized unmixing may increase the enhancement of small absorption features. To illustrate how the discrimination of ground components is influenced by the standardization, figure 3 

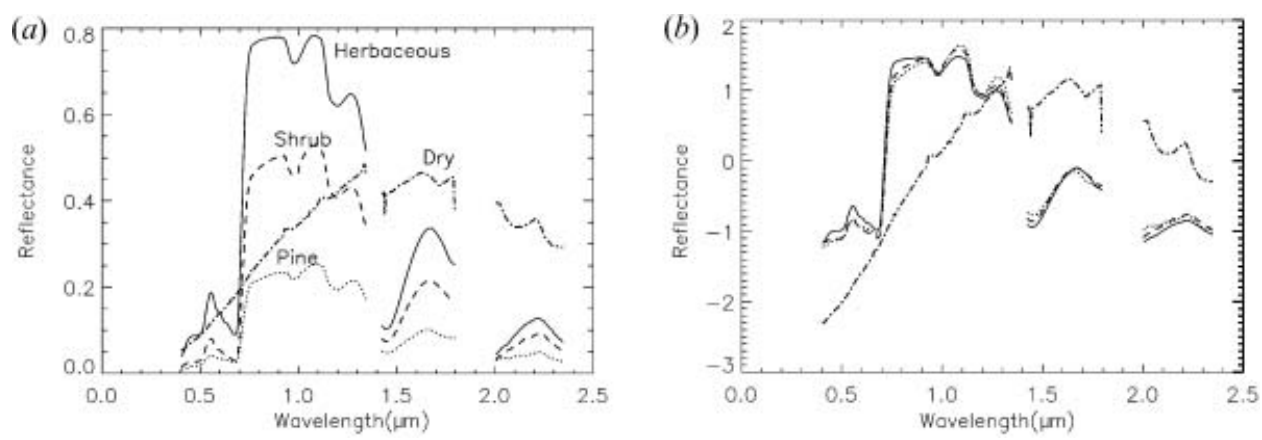

Figure 3. Four different vegetation types: green herbaceous (Asphodelus albus), green shrub (Euphorbia characias) with some shade, young dense light green forest (Pinus brutia) and dry shrub (Arbustus unedo). Absolute reflectance (a), standardized reflectance (b).

compares different green vegetation spectra, selected from the MedSpec database (see §2.6), with their corresponding standardized spectra.

Logically, differences in architecture of the various plant canopies (grass, shrub and forest) cause different shade proportions. However, after performing the standardization, only one vegetation spectrum may be sufficient to represent the spectral variability of the green vegetation components. Similar results have been obtained analysing real images. This proves the effectiveness of standardized SMA to represent the spectral variability of green vegetation types using as few as one single vegetation EM despite the variable structure of scene cover types. Figure 4 shows another example corresponding to four spectrally separable soils. The standardization removes most of the spectral differences associated with soil texture (i.e. the grain aggregation) while retaining the inherent features related with its composition.

Figure 5 shows the distribution, in the principal component (PC) feature space, of a set of 260 spectra extracted from the MedSpec database to represent the dominant vegetation species, soils and rocks. In order to address the influence of the soil background, we have included in the analysis two samples corresponding to the same green broad leaf placed on two different soil backgrounds, a very dark soil (D) and a very bright one (B).
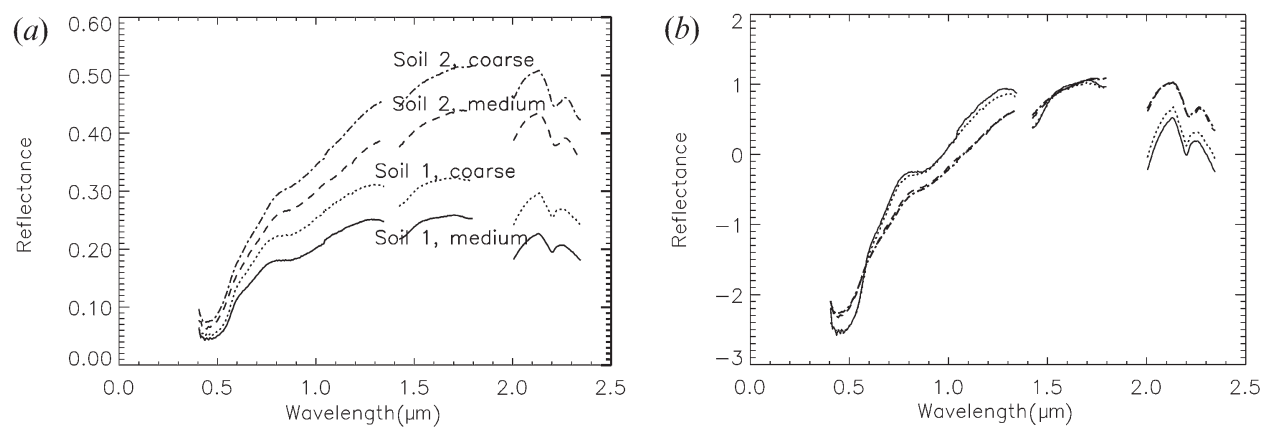

Figure 4. Four different soil types: silty dark reddish soil (type 1) and brown reddish Karst filing soil (type 2), with two different types of aggregation, coarse and medium. Absolute reflectance $(a)$, standardized reflectance $(b)$. 

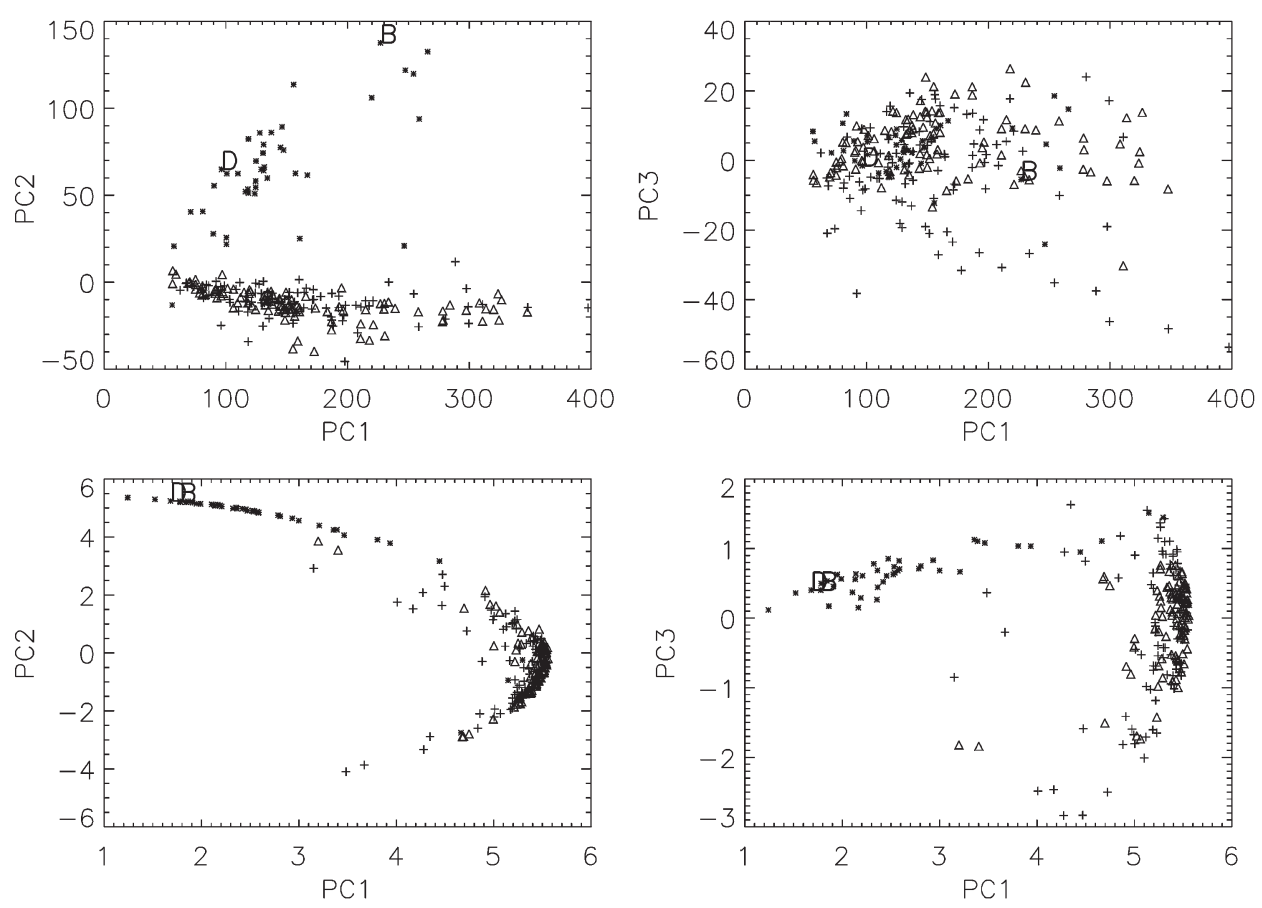

Figure 5. Distribution, in the abstract space of the primary PCs, of 260 MedSpec spectra representing the dominant cover classes of the Mediterranean areas. Different symbols were used to label vegetation (*), soil ( 4 ) and rock (+). The positions of B and D samples have been marked.

We can observe that the spectral features of the green vegetation are highly influenced by the optical properties of the soil background. Nevertheless, the use of standardized units considerably normalizes this influence (i.e. the positions of B and $\mathrm{D}$ are almost coincident). The analysis has also shown that broad land cover classes such as canopy areas, desert and urban areas are more easily discriminated using standardized units. Consequently, standardized unmixing may simplify the EM submodels because it reduces the model sensitivity to the natural variability of EM spectra, calibration errors, topography or differences in illumination conditions.

\subsection{The model performance: the colinearity factor}

The model performance is usually assessed in the literature from the residual spectrum (e.g. rms, maximum residual or the quadratic form $\chi^{2}$ (equation (4)). We have developed a new and alternative criterion that has been demonstrated to be very robust and faster than the residual one, since it reduces the dimensionality of the problem. We will now briefly summarize its formulation. Let us consider a mixed pixel with reflectance spectrum $\boldsymbol{r}$, and a subset of $c$ potential EMs represented by the column vectors $\boldsymbol{E}_{1}, \ldots, \boldsymbol{E}_{c}$. Let $\boldsymbol{M}$ be a $p \times n$ matrix $\boldsymbol{M}=\left\{\boldsymbol{E}_{1}, \ldots, \boldsymbol{E}_{c}, \boldsymbol{r}\right\}$, where $n$ is the number of spectral bands and $p=c+1$. A $p \times p$ matrix $\boldsymbol{A}$ is constructed using the expression:

$$
\boldsymbol{A}=\boldsymbol{M} \cdot \boldsymbol{M}^{T}
$$

If the pixel is a mixture of the $c$ considered EMs, the last column vector of matrix $\boldsymbol{M}$ 
will be spanned by the other column vectors, and hence $\boldsymbol{M}$ will be a singular matrix. The same reasoning applies to matrix $\boldsymbol{A}$. Consequently, the aim is to determine the set of $c$ EMs that maximizes the 'singularity' of matrix $\boldsymbol{A}$. Since $\boldsymbol{A}$ is a symmetric positive definite matrix, it is possible to apply a Cholesky decomposition, i.e. to determine an upper triangular matrix $\boldsymbol{L}$ such that:

$$
\boldsymbol{A}=\boldsymbol{L} \cdot \boldsymbol{L}^{T}
$$

The solution, referred to as 'taking the square root' of the matrix $\boldsymbol{A}$, is expressed by the following equations (Nash 1990, Press et al. 1992):

$$
\begin{gathered}
L_{i i}^{2}=\left(a_{i i}-\sum_{k=1}^{i-1} L_{i k}^{2}\right) \\
L_{j i}=\frac{1}{L_{i i}}\left(a_{i j}-\sum_{k=1}^{i-1} L_{i k} L_{j k}\right) \quad j=i+1, i+2, \ldots, c+1
\end{gathered}
$$

To simplify the computations, a normalization transformation can be applied on all EM spectra, in such a way that $a_{i i}=1(i=1,2, \ldots, c)$. All except the last column of matrix $\boldsymbol{L}$ need to be determined only once for each EM subset. It turns out that the pixel reflectance will be a linear combination of the EMs only if the term of the triangular matrix $L_{p p}$ (or equivalently, $L_{p p}^{2}$ ) is equal to zero. We will refer to $L_{p p}^{2}$ as the colinearity factor, since it indicates to what extent $r$ is expressed as a linear combination of the candidate subset (i.e. lower values indicate higher confidence that the submodel EMs explain physically correct the mixed spectral signature). The colinearity factor is equal to the Euclidean distance from $\boldsymbol{r}$ to the subspace spanned by the EMs, and is similar to the rms residual of the leastsquare solution as obtained without the sum-to-one constraint. In fact, a low residual implies a high degree of colinearity. Several tests on simulated mixtures with varying levels of noise have shown that the colinearity factor is as powerful as methods based on the residuals to identify the best EM subset, though it is considerably faster.

Logically, the colinearity factor does not take into account the problem of negative abundances, i.e. when the orthogonal projection in the features space lies outside of the EM's polyhedron. Nevertheless, in order to incorporate the sum-toone constraint to the solution it is possible to use, instead of the matrix $\boldsymbol{M}$, a matrix $\boldsymbol{M}$ of dimensions $p \times n+1$ as obtained by adding to matrix $\boldsymbol{M}$ a column vector of value $w$. This value controls the relative weighting of the sum-to-one condition compared with the model performance. In addition to a high computational efficiency, the colinearity factor presents other advantages:

1. It is not biased, i.e. it is zero only if $\boldsymbol{r}$ is a perfectly linear combination of EMs, whereas the higher the colinearity factor, the lower the level of reproduction of $r$.

2. It is well-defined, i.e. invariant respect to a change of the EMs order.

3. The number of EMs is usually unknown and the problem thus becomes to unmix the given compositions into a number of EMs supported by the dimensionality of the data. The colinearity factor enables a direct comparison between submodels having a different number of EMs. Increasing the number of EMs by one usually translates into a lower colinearity factor, since the 
accuracy of the reconstruction tends to increase with the number of freedom degrees. Nevertheless, this increase of model performance may translate into more unstable fractions due to progressive lowering of spectral contrast between EMs, thereby causing higher fractional errors.

\subsection{An expert system to select the best submodel}

An efficient and accurate method is needed to determine the EMs making up each reflectance pixel. An expert system, which does not require prior knowledge of the study area, was developed with this aim. The algorithm basically ties together modelling errors and fractional errors, and makes an automated decision to assign each constituent material to its optimum EMs subset. The procedure is composed of three steps (see figure 6): (1) The colinearity factor criterion is applied to all submodels in order to preselect the most probable candidates, i.e. a small number of submodels presenting the lowest colinearity factor; (2) fractional errors $\left(\varepsilon_{\text {neg }}\right)$ and modelling errors $\left(\varepsilon_{r}\right)$ are used to identify the most likely 2-, 3- and 4-EM subsets, and (3) the three best subsets are compared using an expert system in order to select a single subset. We now describe steps 2 and 3 .

2.4.1 Decision between subsets with the same number of EMs. This step involves the comparison among preselected submodels with the same number of EMs. $\varepsilon_{\boldsymbol{r}}$ is assessed either from the colinearity factor, the rms or the maximum residual, depending on the user specifications, whereas $\varepsilon_{\text {neg }}$ is calculated from the summation of the abundances that lie outside the interval $[0,1]$, i.e.

$$
\varepsilon_{\text {neg }}=\frac{1}{2} \sum_{k=1}^{c}\left(\left|\hat{f}_{k}\right|-\hat{f}_{k}\right)+\frac{1}{2} \sum_{k=1}^{c}\left\{\left|1-\hat{f}_{k}\right|-\left(1-\hat{f}_{k}\right)\right\}
$$

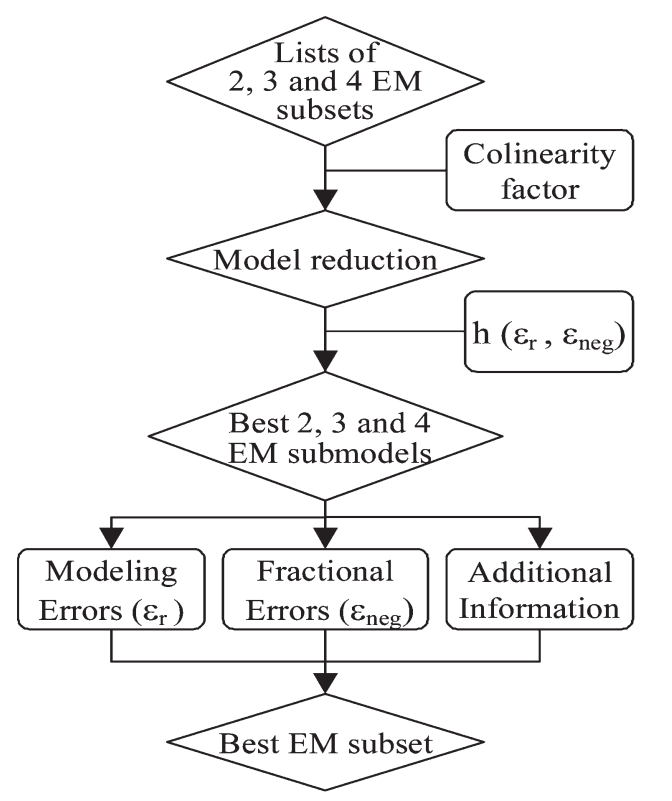

Figure 6. Flowchart of the expert system used to select the EM subset. 
where $\hat{f}_{k}$ represents the estimate of the true proportion $f_{k}$. The main difficulty consists of weighting appropriately both types of error in order to express them in comparable units. Based on the observed existence of a strong positive correlation between $\varepsilon_{r}$ and $\varepsilon_{\text {neg }}$, we propose a function, $h\left(\varepsilon_{r}, \varepsilon_{\text {neg }}\right)$, that balances the contribution of both types of error in such a way that the best candidate must minimize the function $h$ :

$$
h\left(\varepsilon_{r}, \varepsilon_{\text {neg }}\right)=\varepsilon_{r} \cdot\left(a+\varepsilon_{\text {neg }}\right)^{b}
$$

where $a$ and $b$ are positive constants. It is obvious that the higher the values of $b$ and $a$, the higher the relative importance of $\varepsilon_{\text {neg }}$ in the decision. To gain an insight into the problem, let us compare two submodel candidates, $i$ and $j$, and define the terms $\Delta \varepsilon_{r}=\varepsilon_{r(i)}-\varepsilon_{r(j)}$ and $\Delta \varepsilon_{\text {neg }}=\varepsilon_{\text {neg }(i)}-\varepsilon_{\text {neg }(j)}$. Let us consider the non-trivial case, i.e. when $\Delta \varepsilon_{\text {neg }}$ and $\Delta \varepsilon_{r}$ have different sign, e.g. $\Delta \varepsilon_{r}>0$ and $\Delta \varepsilon_{\text {neg }}<0$. The question is to decide if the improvement in model performance of submodel $j$ makes it more suitable than submodel $i$, even though it causes higher fractional errors. By taking increments in equation (18) we have:

$$
\Delta h=b \cdot a^{2} \cdot \varepsilon_{r} \cdot\left(\Delta \varepsilon_{\mathrm{neg}}+\frac{a+\varepsilon_{\mathrm{neg}}}{b} \frac{\Delta \varepsilon_{r}}{\varepsilon_{r}}\right)^{b}
$$

Hence, model $i$ will be selected only if $-\Delta \varepsilon_{\text {neg }}$ overweighs $\frac{a+\varepsilon_{\text {neg }}}{b} \frac{\Delta \varepsilon_{r}}{\varepsilon_{r}}$. Consider an example in which $\frac{\Delta \varepsilon_{r}}{\varepsilon_{r}}=0.3$ (i.e. submodel $i$ performs $30 \%$ better than $j$ ), submodel $j$ presents positive abundances (i.e. $\left.\Delta \varepsilon_{\text {neg }(j)}=0\right)$ and appropriate values are assumed for constants $a$ and $b$, e.g. $a=1.0$ and $b=3.0$. With these premises, submodel $j$ is preferred only if $\Delta \varepsilon_{\text {neg }(i)}>0.05$, e.g. if submodel $i$ abundances are as negative as -0.10 for one of its EMs.

2.4.2 Decision between subsets with different number of EMs. In the last step, an expert system selects the best EM subset among the 2-, 3- and 4-EM best candidates. The algorithm chooses first between the 2- and the 3-EM submodels, and then compares the selected submodel with the 4-EM submodel. Results have revealed the advantages of deducing the number of EMs on a per-pixel basis (Brink et al. 2003). For example, a two or three EM solution may be insufficient for many image units because of their high complexity, whereas in relatively uniform sub-areas using as much as four EM submodels would mean there is a high risk of producing mathematically well fitting results that are physically misleading and do not explain the real composition of the pixel.

In general, increasing the complexity of the model (i.e. a 3-EM model compared with a 2-EM model) causes an increase of the model performance (i.e. a reduction of $\varepsilon_{r}$ ) along with an increase of $\varepsilon_{\text {neg. }}$. Thus the idea of a mixed criterion is that a 2-EM model is preferable when it performs similarly to a 3-EM model (in terms of both errors, $\varepsilon_{r}$ and $\varepsilon_{\text {neg }}$ ), whereas a 3 -EM model is preferred if it performs significantly better. The algorithm computes both the difference between 2- and 3EM 'negative errors' (i.e. $\Delta \varepsilon_{\text {neg }}=\varepsilon_{\text {neg } 3}-\varepsilon_{\text {neg2 } 2}$ ) and the percentage of improvement in the modelling error (i.e. improvement $[\%]=100 \frac{\Delta \varepsilon_{r}}{\varepsilon_{r 2}}=100 \frac{\varepsilon_{r 3}-\varepsilon_{r 2}}{\varepsilon_{r 3}}$ ). $\Delta \varepsilon_{\text {neg }}$ determines the improvement in the modelling error that is necessary to compensate for this decrease of negative errors. An example of mixed criterion is given by the following pseudocode: 
Case of
1: $\Delta \varepsilon_{\text {neg }}<-0.03$
$\rightarrow 3$-EM
2: $\left(-0.03<\Delta \varepsilon_{\text {neg }}<0.03\right)$
$\rightarrow$ if improvement $<10 \%$ then 2-EM else 3-EM
3: $\left(0.03<\Delta \varepsilon_{\text {neg }}<0.08\right)$
$\rightarrow$ if improvement $<20 \%$ then 2 -EM else 3 -EM
4: $\left(0.08<\Delta \varepsilon_{\text {neg }}<0.15\right)$
$\rightarrow$ if improvement $<30 \%$ then 2 -EM else 3 -EM
5: $\left(0.15<\Delta \varepsilon_{\text {neg }}<0.20\right)$
$\rightarrow$ if improvement $<40 \%$ then 2 -EM else 3 -EM
6: $\Delta \varepsilon_{\text {neg }}>0.2$
$\rightarrow 2-\mathrm{EM}$

According to these criteria, in case of non-negative abundances (i.e. $\left.\Delta \varepsilon_{\text {neg }}=\varepsilon_{\text {neg } 1}=\varepsilon_{\text {neg } 2}=0\right)$ a 2 -EM model is preferred when improvement $<10 \%$ and a 3-EM model otherwise.

\subsection{Updating the segmentation}

Stratifying the images may improve identifications of specific compounds and is the basis of the variable unmixing strategy. Multispectral information (e.g. reflectances, PCs, NDVI), unmixing results (e.g. modelling errors, abundances) and auxiliary data (e.g. land cover, land use, soil type) may assist in revealing the structure of the area and allow its partition into characteristic zones with user-specified attributes. The segmentation frame (see figure 7) allows for simple mask definition using logical relational operators or brightness histogram thresholding based upon multiband open files. Simple masks define compound masks through the concatenation of Boolean operators combined at will.

In addition, the interface allows for:

1. Interactive selection of regions of interest (ROI).

2. Definition of spectral class boundaries using an interactive 2-dimensional scatter plot. Masking and scattergram analysis are performed at the level of individual subunits, increasing thus the benefit of the hierarchical assessment.

\subsection{Estimation of key EMs and submodel creation}

The categorization of the scene is especially helpful in estimating image EMs in userdefined areas. The Spectral Library Sheet allows the extraction of image EMs using different tools such as non-supervised clustering, PCA-based algorithms or the use of texture information to seek for homogeneous areas (e.g. water bodies, dense forests, bare soil). Even though the above methods are limited to address external EMs, i.e. materials not fully occupying a pixel, this weakness may be alleviated by spectrally matching the image EMs with a spectral library, which moreover provides them with a physical interpretation. 


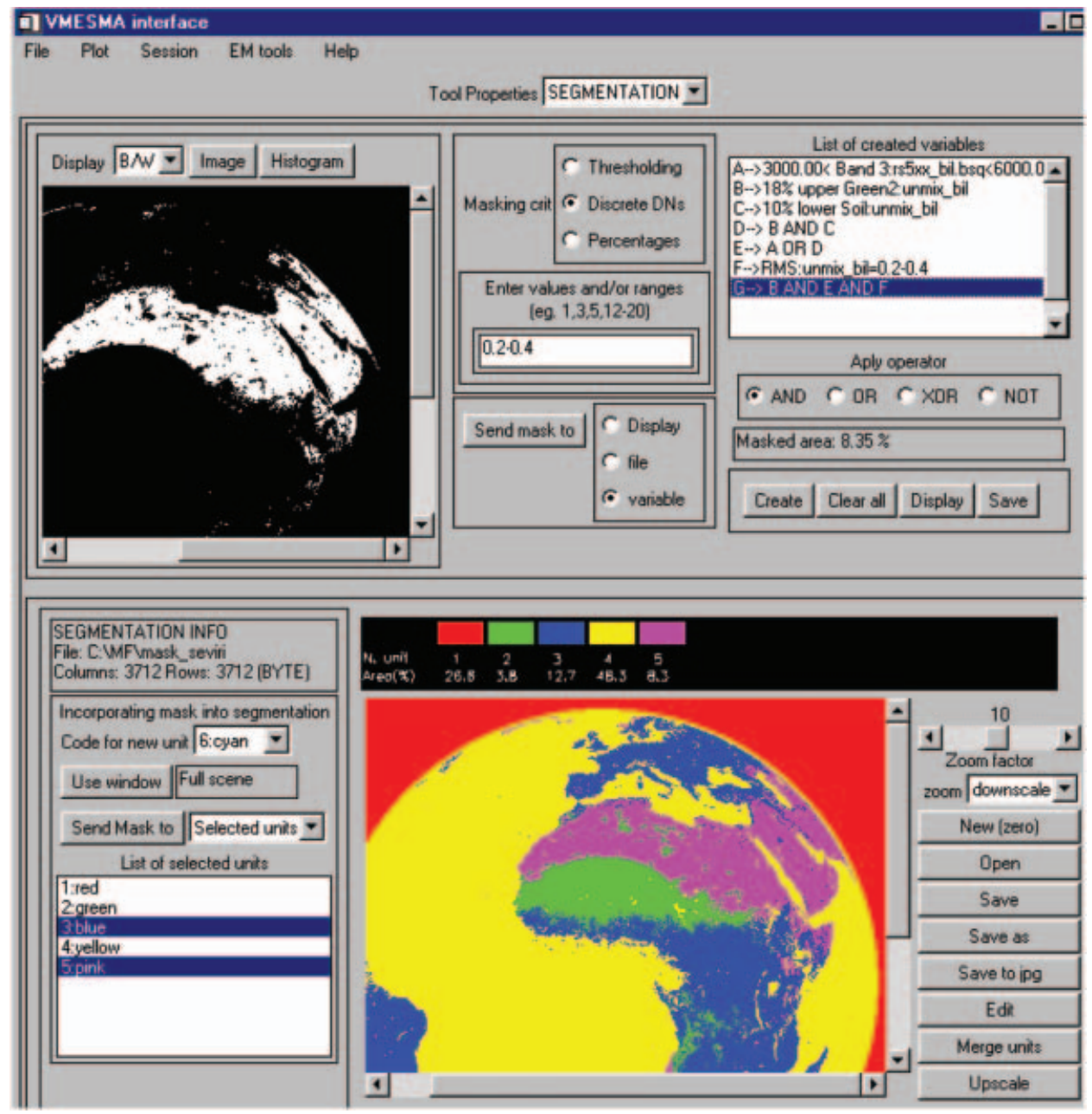

Figure 7. The Segmentation sheet.

The spectral matching function compares the specified spectrum against each spectrum (reference EM) of a regionally specific spectral database, namely MedSpec, the Mediterranean Spectral database (Preissler et al. 1998). This database contains about 1500 visible to SWIR spectra and data acquired during many field campaigns in the Mediterranean countries.

Several different methods are used to enhance the discrimination, including spectral angle (Boardman and Kruse 1994), Euclidean distance in standardization units and band-shape fitting (Kruse 1988). The final decision relies on the combined scores of the considered metrics along with the visual examination of the matching.

Usually EM subsets with only a limited number of components (2-4) should be sufficient to model a pixel. Otherwise noise would exert a significant influence on the proportion estimates of the lowest contrast materials. A function enables the user to create and edit the 2-, 3- and 4-EM combinations by selecting them from the current library. Additional constraints may be added, e.g. to prevent EMs showing small spectral separability occurring together. 
Taking decisions about model creation may be useful in order to reduce the model set to a smaller, more appropriate one, thereby saving computational time and improving the final results (Roberts et al. 1998). Models that account for less than a prescribed percentage, are too fragmented, or that cause unrealistic proportions, can be interactively removed.

\subsection{Defining the covariance matrix}

The VMESMA algorithm requires specifying the covariance matrix of the observations, $\boldsymbol{V}$ (see equation 4). The root squares of the diagonal elements of $\boldsymbol{V}^{-1}$ constitute the weighting factors of each individual band. In general, channels should be prevalent that are more uncorrelated, present higher radiometric accuracy and enhance the contrast between EMs. Simulations using synthetic data have shown that this weighting scheme improves the accuracy and efficiency. For example, a preliminary study has shown that the estimation of carbonates and clay is increased using higher weights for channels corresponding to their major absorption bands in the SWIR (i.e. [2200, 2450] nm). The suitability of this region to unmix green vegetation, litter and soil in arid environments was also demonstrated (Asner and Lobell 2000).

The use of weighting factors to incorporate a priori knowledge into the inversion unmixing algorithm is even more critical when the analysis combines channels with different characteristics, e.g. thermal channels and NDVI (DEMON-II 1999). The different potential of data from the spectral and angular domains to retain information of ground properties in different applications is a critical aspect in the development of operational algorithms for new sensors (Privette et al. 1996, GarcíaHaro et al. 2002).

\subsection{Combined use of different levels of detail}

The large quantities of data associated with multi- and hyperspectral images may cause severe problems in data handling and storage. To reduce them and speed up the computation capabilities, the algorithm includes a compression tool that performs a data quantity reduction by a desired factor. The use of small images brings previously slow computation up to interactive speeds. The compression scheme subdivides the data into square blocks $($ e.g. $3 \times 3$ or $5 \times 5)$ and takes a single pixel value from the block centre.

The first steps of the process - EM submodel creation, segmentation of the area and identification of unmodelled EMs - are thus performed using smaller images, whereas the level of data quality is increased in the successive unmixing iterations. Several tests have shown that the unmixing outcomes, such as mean and standard deviation of abundances, submodel percentages, rms and fraction histograms, are almost invariant with respect to this compression scheme (e.g. the fractions changed typically less than $0.5 \%$ on average).

When using hyperspectral data, a similar compression scheme is also possible in the spectral domain (Ben-Dor and Banin 1994, Kemper and Sommer 2002).

\section{$2.9 V M E S M A$ resources}

The software is a modular environment that is cross-platform and robust. The frame is implemented in Interactive Data Language, IDL, and fully supported by a 
Graphical User Interface (GUI), which provides a graphical means of interacting with menus and data. System memory usage is optimized by breaking the images into manageable pieces (e.g. blocks of rows or spatial subsets) before being processed in memory in order to alleviate the high demand in random access memory. The most time demanding computations have been implemented in $\mathrm{C}++$ and Fortran95 languages.

The frame is organized into four Property Sheets: 'Reflectance image', 'Library', 'Segmentation' and 'Unmixing' (see examples in figures 2 and 7), which allows common processing problems to be addressed with the necessary flexibility. Although not shown for the sake of brevity, they include advanced methods to improve the likely accuracy of estimating proportions, such as: (i) an image restoration method, which corrects the effects on the fractions due to the spatial degradation of the signal caused by the scanner Point Spread Function (PSF); and (ii) a contextual method that relies on estimating fractions in surrounded pixels to improve the selection of local EMs (e.g. less fragmented submodels). The interface also includes batch-processing of archived datasets and session back-up facilities for making the unmixing process repeatable.

\section{Application: mapping residual tailings sludge after a mining accident}

The application of VMESMA is demonstrated in a case study on contamination mapping after a mining accident in Spain. The collapse of the tailings pond in Aznalcóllar in April 1998 had left several thousands of hectares in the Guadiamar floodplain contaminated with pyritic sludge and high concentrations of trace metals. Pyrite oxidization is one of the most acid-producing natural weathering processes, in which trace metals are mobilized and released into the river system and the groundwater. The iron-bearing secondary minerals are iron-rich and hydroxyland/or water-bearing, which makes it possible to identify them on the basis of their diagnostic spectral reflectance features (Swayze 2000). Thus, one of the objectives of this case study was identification of residual contamination and monitoring of the oxidation process using hyperspectral data. The ongoing remediation work and intense geochemical processes caused strong interannual variations, which make high demands on the flexibility of the analysis tool with respect to image analysis and EM selection, and are thus an appropriate test case for VMESMA.

\subsection{Study area}

The study area is situated approximately $40 \mathrm{~km}$ west of Seville, Spain (figure 8). After a collapse of a tailings dam at the Aznalcóllar Mine on 25 April 1998, four million cubic metres of acidic water and two million cubic metres of toxic sludge with high heavy metal concentrations flooded the Agrio and Guadiamar rivers, endangering the wetlands of the Donana National Park. This national park is one of Europe's biggest wetlands areas and a very important refuge for migrating waterfowl. The toxic sludge had covered an area of 4286 ha, mainly agricultural and marshland. Sludge and contaminated topsoils had been removed within six months after the accident using heavy machinery. Monitoring activities have been started by Spanish institutions to control the possible mobility of residual contaminants due to oxidation processes (Grimalt et al. 1999). 


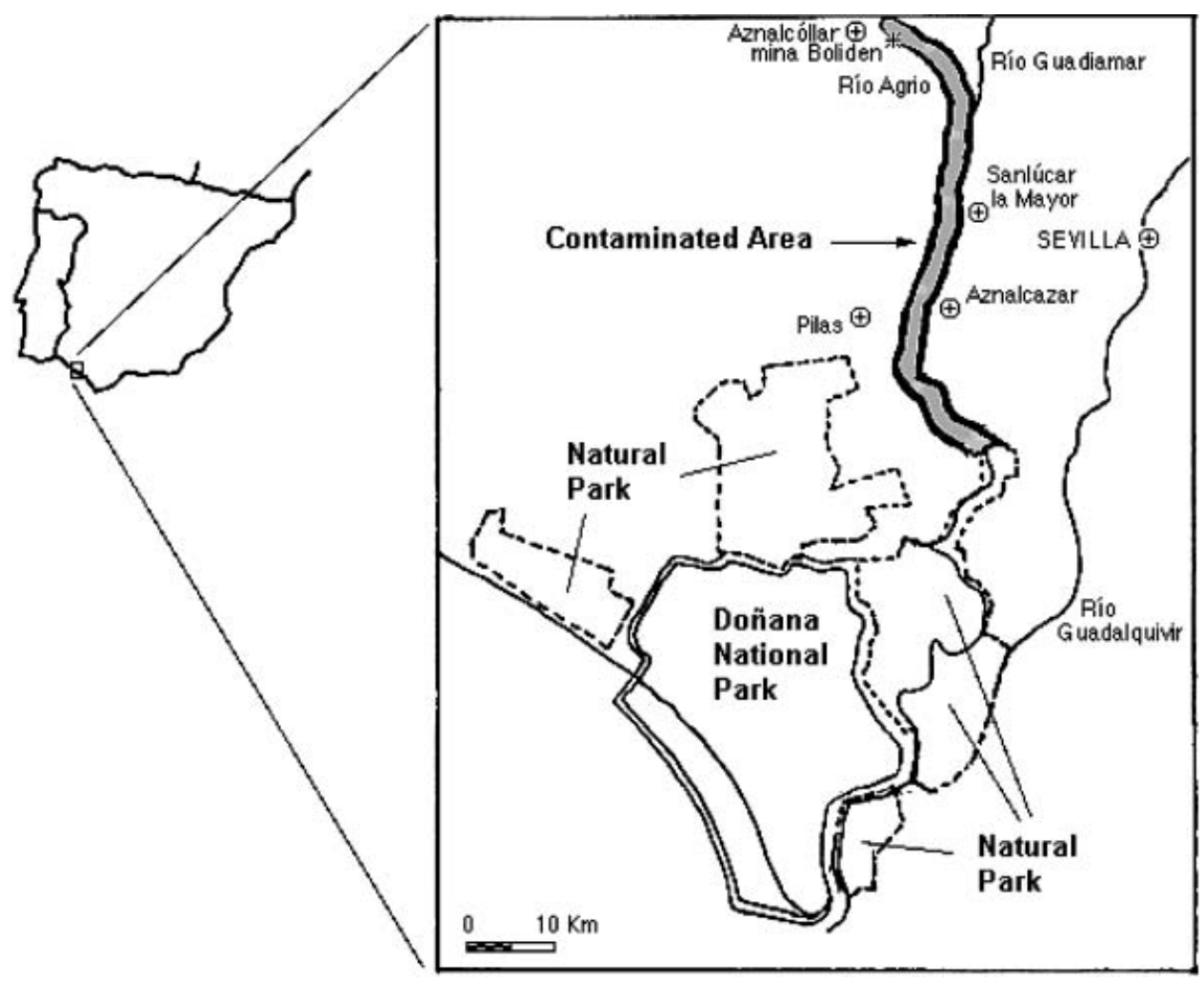

Figure 8. Sketch of the study area.

\subsection{Dataset}

Two extensive field campaigns were carried out in May/June 1999 and June/July 2000. They included airborne imaging spectrometer and concomitant field data. The imaging spectroscopy data were acquired by DLR with the HyMap sensor on behalf of the JRC. The data takes covered the entire contaminated area and were accompanied by a radiometric calibration field campaign. The HyMap system provides 128 wavebands over the range [403, 2480] $\mathrm{nm}$. The data were delivered atmospherically and geometrically corrected by DLR according to the methods described by Richter (1996) and Schlaepfer et al. (1998). Because of the good data quality no further pre-processing was necessary; only 16 bands in the atmospherically affected areas of the spectrum were excluded from further analysis.

Nearly simultaneously, representative sites along the affected river catchments were selected and spectrally characterized. Spectral measurements taken using GER S-IRIS and ASD Fieldspec II resulted in a VIS to SWIR highresolution library hierarchically organized of rocks, soils and vegetation, close related to their physiographic context. The spectra were integrated in MedSpec database. Furthermore, a detailed soil sampling was carried out for geochemical laboratory analysis in order to characterize the contamination level of the soils. 


\subsection{Results and discussion}

Our purpose is to extract the highest amount of systematic variability that is related to our interests (i.e. sludge and sludge derivatives), while minimizing the effect of the variability that is related to the other properties present in the area (soil, vegetation, water bodies). The structure of VMESMA allows an iterative analysis of the image, which is of utmost importance in this study. In a first step the VMESMA segmentation tool $(\$ 2.5)$ is used to separate non-affected areas from the contaminated areas based on GIS information about the maximum extent of the sludge.

In the next step the EM selection capabilities of VMESMA were used for the identification of appropriate EMs (\$2.6). The selection of EMs has to consider the variability of the occurring surface materials, the spatial and spectral resolution of data and the thematic purpose of the study. For the extraction of the residual sludge signal it was necessary to separate sludge related spectral information from other 'background' information. Using the automated EM selection, followed by the spectral matching capacity of VMESMA, spectra of green and dry vegetation and two different soils were selected from the spectral database as background information (figure9). A spectrum of stacked sugar beet leaves was used to represent the green vegetation EM whereas the dry vegetation EM was measured over a stack of dry grass. The brighter soil had a fine texture and a yellowish red colour (Munsell value 5YR 5/6), the absorption at about $1.0 \mu \mathrm{m}$ is indicative of certain amounts of iron oxides and the absorption at $2.2 \mu \mathrm{m}$ is typical for clay minerals. The darker soil has an intermediate structure and a brown colour (Munsell value 7.5YR 5/3). This soil does not exhibit strong iron absorption, but has a distinct absorption caused by clay minerals.

To select sludge EMs we had to consider the potential secondary minerals of pyrite, such as jarosite, copiapite, ferrihydrite and goethite. However, for the 1999 images, the oxidation was limited to areas with a constant water supply and for the major part of the contaminated area no considerable oxidation took place. Thus, a single spectrum of pure sludge was sufficient to represent the sludge variability. This result was also confirmed by the automated EM selection, which also identified a
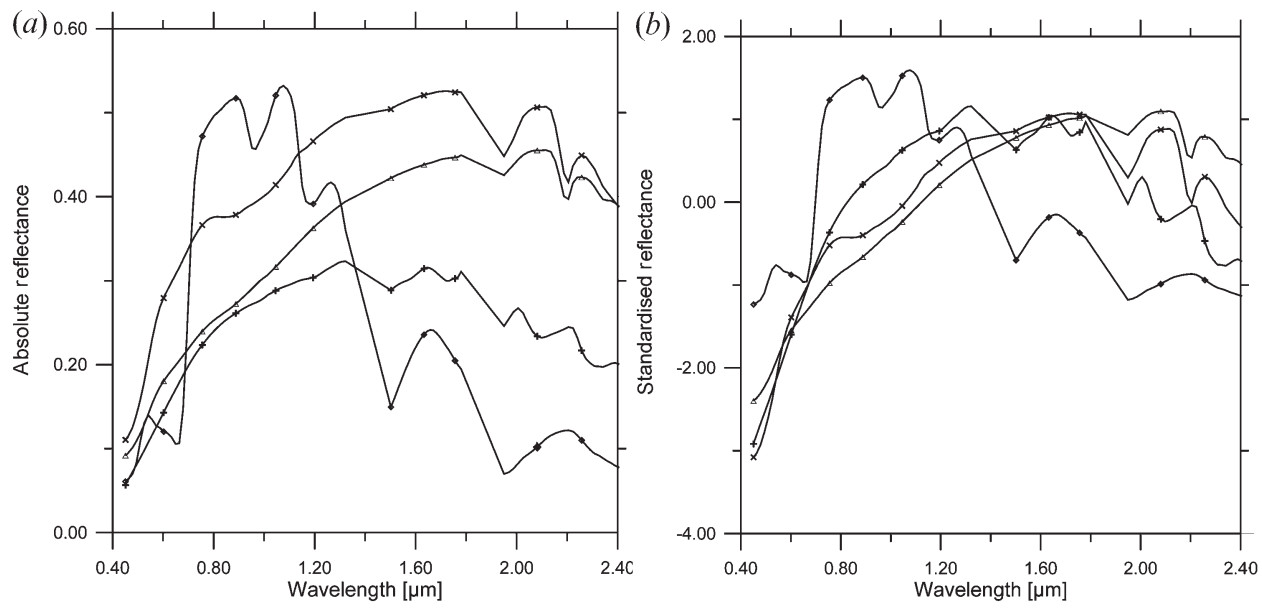

Figure 9. Background EM: green vegetation $(\diamond)$, dry vegetation $(+)$, soil $1(\Delta)$, soil $2(\times)$. Absolute reflectance $(a)$, standardized reflectance $(b)$. 



Figure 10. Sludge and secondary mineral EM: pyritic sludge $(\diamond)$, yellowish jarosite dominated crust $(+)$, bright gypsum crust $(\Delta)$. Absolute reflectance $(a)$, standardized reflectance $(b)$.

sludge spectrum besides soil and vegetation spectra. In the year 2000 other sludge related spectra had to be taken into consideration, because field and image assessment confirmed that the oxidation processes had produced various crusts on the soil surface. During the second field campaign, spectral measurements of the most abundant crusts were recorded, from which the EMs were selected (see figure 10). Figure 10 illustrates another advantage of VMESMA for this case study. The standardization strongly enhances the absorption features of the sludge spectra compared to the reflectance spectra. In particular, the pyritic sludge, which in reflectance is almost featureless, has a more distinct shape after standardization.

In the first unmixing step, the four background spectra were used to unmix the entire scene. Although a maximum of four EMs per pixel was allowed, only in less than $0.5 \%$ of the cases as many EMs were used. In the majority $(75 \%)$ two EMs were sufficient to model the scene. The rms obtained after the first unmixing clearly separated the sludge-affected areas with a high rms from the non-contaminated areas. A good agreement was found between these areas and the maximum sludge extent. In areas with a higher rms the residuals also clearly reflect the lack of another EM. On the other hand, in most of the non-affected areas, the proposed EMs were sufficient, which is illustrated by low rms and featureless residuals (figure 11). Prior to the next unmixing step, the segmentation tool was used to stratify the image. The abundances outside the affected area were disregarded, and in the affected area, an rms threshold was used to separate sufficiently well-modelled areas from areas most probably affected by sludge.

The resulting sludge abundance map for June 1999 (figure 12) shows that the sludge abundances were still very high, with an average abundance of 0.51 . The results were obtained with only one sludge EM. In fact, tests with additional sludge EMs were not successful. This is a clear indicator that at this point in time, 13 months after the accident, oxidation of pyrite did not yet reach a high intensity and consequently it was possible to map the sludge using only the pyrite sludge EM. The estimated sludge distribution is in good agreement with the field observations and the results of the geochemical analysis, and reflects the discontinuous distribution pattern caused by the mechanical clean up. 

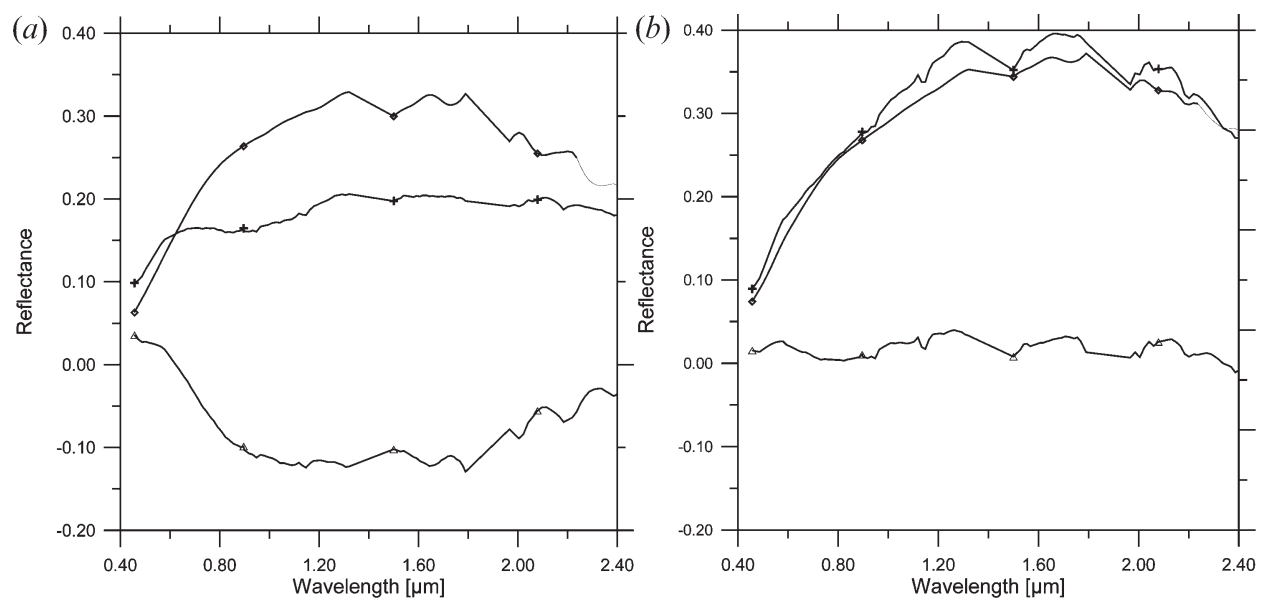

Figure 11. Comparison of original $(+)$ and modelled $(\diamond)$ spectra and residuals $(\Delta)$. Pixel in sludge affected area $(a)$ and pixel in non-affected area $(b)$.

The same unmixing strategy was applied for the data collected in 2000. However, the situation had changed significantly. The remediation activities proceeded with a second cleaning phase in combination with fixation of the trace metals. This was achieved by augmentation of the $\mathrm{pH}$ level of the soil through addition of lime-rich material. At the time of the second field and flight campaign the work was still ongoing. In many areas, particularly in the northern part, efflorescent crusting could be observed. According to Nordstrom (1982), these crusts are most commonly formed during dry periods when evaporation promotes the rise of subsurface water to the uppermost soil surfaces by capillary action. As the water reaches the surface it becomes progressively more concentrated and finally precipitates various salts in efflorescence. The formation of these iron sulphate salts is an intermediate step, which precedes the precipitation of more common insoluble iron minerals such as goethite and jarosite. In order to account for this change in surface composition two new EMs representing these efflorescent crusts were included in the SMA modelling (see figure 10).

The abundance map for July 2000 shows a considerable reduction of areas with sludge EM abundances compared to the abundances obtained for June 1999 (figure 12). The reduction was achieved by the second remediation process initiated by the local authorities. However, in areas where the remediation was not finished the oxidation of the residual sludge had started, resulting in higher abundances of secondary minerals. Major problematic areas are found in the gravel pits at Sobarbinas and in the riverbed of the Agrio River at the confluent of the Agrio and Guadiamar rivers, where the sludge wave contaminated the wide floodplains. In this area, jarosite was found to be abundant. It is formed under dryer conditions according to the reaction sequence explained by Nordstrom (1982). The patches of gypsum are restricted to shallow depressions, in which more water gathered after rainfalls and the humidity was sufficient for the formation of gypsum when the water evaporates. However, below these thin surface crusts, only a few millimetres thick, secondary minerals are found. Thus, the presence of gypsum is on the one hand an indicator of buffering of acidity by the distributed material Ca-rich material; on the other hand it shows that there is still residual sludge in the soil, which produces acidity. 

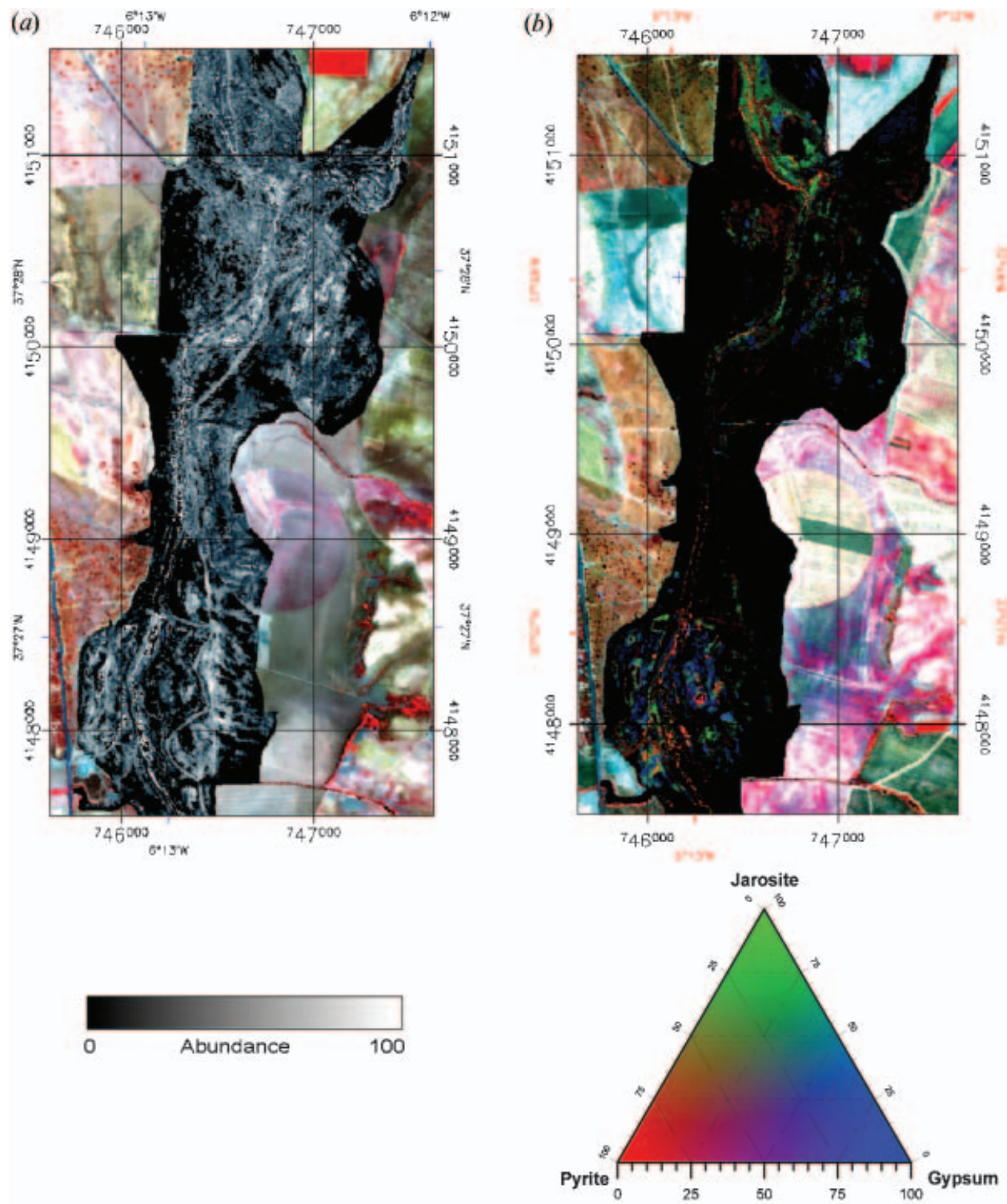

Figure 12. (a) Sludge abundance map 1999. The affected area (black) superimposed on the HyMap false colour image for better orientation. Sludge abundance within the affected area is scaled from zero (black) to one (white). (b) Mineral abundance map 2000. The mixtures of the different EMs can be derived from the colour coded ternary diagram. Red is the pyrite EM, green is the jarosite EM, blue the gypsum EM.

For comparison, the abundances were also estimated using the conventional approach with a fixed EM set including a shade EM. Consequently, the image from 1999 was unmixed using the four background EMs, the sludge EM and shade. For retrieval of absolute abundances the shade EM was removed and the remaining EMs were rescaled as outlined in $\$ 2.2$. However, the increased number of EMs in combination with rescaling due to the shade EM produced a high number of negative fractions. For the conventional approach $63.1 \%$ of the pixels are negative in 
at least one fraction and $37.1 \%$ are more negative than -0.1 ; using the VMESMA approach only $0.01 \%$ of negative pixels occur.

In addition, using a fixed EM set overestimates the area in which sludge is present, on the one hand, and on the other hand sludge abundances in contaminated areas are underestimated due to the spectral similarity between sludge and shade.

\section{Conclusions}

The degree of validity of spectral mixture analysis (SMA) is limited by the presence of multiple scattering, directional effects and difficulties of addressing the spectral EMs in the scene. In this paper, we have developed a methodology that extends the possibilities of conventional SMA. This method is aimed at improving understanding and modelling of surface optical properties in the EM selection. EMs are chosen to reflect the actual surface conditions in the scene, while at the same time maximizing the spectral contrast, which is necessary to obtain improved abundances. The basis is a hierarchical subdivision of the image for addressing the variations of the level of complexity between the various image sub-areas along with an iterative feedback process to focus the analysis on problematic areas.

VMESMA requires expert monitoring for guided injection of prior spatial and spectral knowledge from different data sources, including the assessment of previous unmixing results. While potentially a weakness, the method is potentially better suited to meet the research requirements and to reduce model sensitivity to nonlinear/directional effects and data inaccuracies. The streamlined use of a priori knowledge in the unmixing process is considered the best option, if not necessary, when different submodels tend to model the same areas equally well, which is very common with low spectral resolution data. In addition, VMESMA mitigates possible unmixing errors when key spectra are missing in the library or can be found only in small parts of the scene (local EMs).

The VMESMA approach offers also new contributions for fulfilment of unmixing analysis, including methods for dynamic identification of the optimum EM subset and an alternative solution, namely standardized unmixing. This solution reduces EM spectral variability due to shade and brightness variations attributed to illumination and topography. Although this reduction can be a serious drawback to distinguish subcanopies with different structures, such as crop varieties and forest types, the solution may outperform conventional unmixing when the property is masked by external factors (e.g. illumination) and canopy shade. Furthermore, standardized unmixing is potentially more suited for hyperspectral applications since it may enhance small absorption features.

The results of VMESMA experiments, compared to those of a conventional procedure, show that the new method identifies more clearly the spectral signal associated to all scene components and significantly reduces the modelling error. In particular, the potential of VMESMA to map sludge residual has been demonstrated on hyperspectral airborne data. In summary:

1. The iterative approach starting with a simple EM configuration of background materials allowed delineation of the affected area from the rms values, which was congruent with the GIS layers of the affected area.

2. The standardization of spectra was very helpful for detection of sludge, which is a very dark and almost spectral featureless material. First, it was possible to use EM models without shade, which caused problems in separation of shade 
and sludge. Second, the standardization enhanced the wide absorption features of sludge, while letting different types of green vegetation appear so similar that a single vegetation EM is sufficient to cover their variability.

3. A comparison between the sludge abundance obtained in 1999 and the oxidation products obtained in 2000 showed that imaging spectroscopy is able to follow the full process of pyrite oxidation: the initial pyrite could be identified in 1999 mapping the oxidation potential. In 2000, the complex weathering leads to an efflorescence of easily dissolved salt crusts and more stable secondary minerals, which could be differentiated and mapped using VMESMA.

\section{Acknowledgments}

This work has been supported by the EC Joint Research Centre by a post doctorate grant awarded to J. García-Haro and a PhD grant awarded to Th. Kemper. J. García-Haro currently has a research position 'Ramón y Cajal' from MCyT, Spain. Special thanks are due to the anonymous reviewers for their extremely helpful suggestions.

\section{References}

Accioly, L., Huete, A.R. and Batchily, K., 1998, Using mixture analysis for soil information extraction from an AVIRIS scene at the Walnut Gulch Experimental Watershed-Arizona. Airborne VISIBLE/Infrared imaging spectrometer (AVIRIS). In Airborne Geoscience Workshop Bibliographies, 12-14 January 1998 (Pasadena, CA: JPL Publications), CD-ROM.

Adams, J.B., Smith, M.O. and Johnson, P.E., 1986, Spectral mixture modelling: a new analysis of rock and soil types at the Viking Lander 1 Site. Journal of Geophysical Research, 91, pp. 8098-8112.

Adams, J.B., Sabol, B., Kapos, V., Filho, R.A., Roberts, D.A., Smith, M.O. and GillesPIE, A.R., 1995, Classification of multispectral images based on fractions of endmembers: application to land-cover change in the Brazilian Amazon. Remote Sensing of Environment, 52, pp. 137-154.

Asner, G.P. and Lobell, D.B., 2000, A biogeophysical approach for automated SWIR unmixing of soils and vegetation. Remote Sensing of Environment, 74, pp. 99-112.

Ben-Dor, E. and BAnIN, A., 1994, Visible and near-infrared (0.4-1.1 $\mu \mathrm{m})$ analysis of arid and semiarid soils. Remote Sensing of Environment, 48, pp. 261-274.

BOARDMAN, J.W., 1989, Inversion of imaging spectrometer data using singular value decomposition. IGARSS 89 Twelfth Canadian Symposium on Remote Sensing, 4, pp. 2069-2072.

Bodrdman, J.W. and Goetz, A.F.H., 1991, Sedimentary facies analysis using imaging spectrometry: a geophysical inverse problem. In Third AVIRIS Workshop, 20-21 May 1991, R.O. Green (Ed.) (Pasadena, CA: JPL Publications), vol. 91-28, pp. 4-13.

BoArdman, J.W. and KruSE, F.A., 1994, Automated spectral analysis: a geological example using AVIRIS data, North Grapevine Mountains, Nevada. In Tenth Thematic Conference on Geological Remote Sensing, 9-12 May, 1994, San Antonio, TX (Ann Arbor, MI: Environmental Research Institute of Michigan), vol. 1, pp. 407-418.

Brink, A., García-Haro, F.J. and Sommer, S., 2003, Mapping soil degradation in Mediterranean ecosystems using a standardised variable unmixing strategy. International Journal of Remote Sensing, in revision.

Camacho-de Coca F., García-Haro, F.J., Gilabert, M.A. and Meliá, J., 2003, Vegetation cover seasonal changes assessment from TM imagery in a semiarid landscape. International Journal of Remote Sensing, 25, pp. 3451-3476. 
Defries, R., Hansen, M. and Townshend, J., 2000, Global continuous fields of vegetation characteristics: a linear mixture model applied to multiyear $8 \mathrm{~km}$ AVHRR data. International Journal of Remote Sensing, 21, pp. 1389-1414.

DEMON-II Project Final Report, 1999, Integrated approaches to desertification mapping and monitoring in the Mediterranean Basin. Internal Report, Joint Research Centre (Brussels: European Commission).

Drake, N.A., Mackin, S. and Settle, J., 1999, Mapping vegetation, soils, and geology in semiarid shrublands using spectral matching and mixture modelling of SWIR AVIRIS imagery. Remote Sensing of Environment, 68, pp. 12-25.

García-Haro, F.J., 1997, Modelización y estimación de parámetros relacionados con la cubierta vegetal en teledetección. PhD thesis, University of Valencia, Spain.

García-Haro, F.J. and Sommer, S., 2002, A canopy reflectance model to simulate realistic remote sensing scenarios. Remote Sensing of Environment, 81, pp. 205-227.

García-Haro, F.J., Gilabert, M.A., Younis, M.T. and Meliá, J., 1995, Análisis de composición espectral (ACE) basado en un modelo de reflectividad para el estudio de la vegetación en una zona semiárida. In Teledetección, usos y aplicaciones, J.L. Casanova and J. Sanz (Eds), pp. 163-170 (Valladolid: Universidad de Valladolid).

García-Haro, F.J., Gilabert, M.A. and Meliá, J., 1996, Linear spectral mixture modelling to estimate amount of vegetation from optical spectral data. International Journal of Remote Sensing, 17, pp. 3373-3400.

García-Haro, F.J., Gilabert, M.A. and Meliá, J., 1999, Estimation of endmembers from spectral mixtures. Remote Sensing of Environment, 68, pp. 237-253.

García-Haro, F.J., Camacho-de Coca F. and Meliá, J., 2002, Retrieval of biophysical parameters using directional spectral mixture analysis. In Proceedings of the 1 st International Symposium on Recent Advances in Quantitative Remote Sensing, 16-20 September 2002, Torrent, Spain (Valencia: Universitat de Valencia), pp. 963-970.

Grimalt, J.O., Ferrer, M. and MacPherson, E., 1999, The mine tailing accident in Aznalcóllar. Science of the Total Environment, 242, pp. 3-11.

Gross, H.N. and SchOTT, J.R., 1998, Application of spectral mixture analysis and image fusion techniques for image sharpening. Remote Sensing of Environment, 63, pp. 85-94.

Hill, J., Smith, M.O., Mehl, W., Lacaze, B. and Sommer, S., 1995, The use of high spectral resolution imaging systems for mapping land degradation patterns in Mediterranean ecosystems. In MAC Europe 91, Final Results Workshop Proceedings, 2-6 October 1994, Lenggries (Frascati: ESA), ESA WPP-88, pp. 31-47.

Kemper, T. and Sommer, S., 2002, Estimate of heavy metal contamination in soils after a mining accident using reflectance spectroscopy. Environmental Science \& Technology, 36, pp. 2742-2747.

KLEIN-GeBBINCK, M.S., 1998, Decomposition of mixed pixels in remote sensing images to improve the area estimation of agricultural fields. PhD thesis, University of Nijmegen (Veenendaal: University Press).

KRUSE, F.A., 1988, Use of airborne imaging spectrometer data to map minerals associated with hydrothermally altered rocks in the northern Grapevine Mountains, Nevada and California. Remote Sensing of Environment, 24, pp. 31-51.

Leblon, B., Gallant, L. and GranberG, H., 1996, Effects of shadowing types on groundmeasured visible and near-infrared shadow reflectances. Remote Sensing of Environment, 58, pp. 322-328.

Mackin S., Settle, J., Drake, N. and Briggs, S., 1991, Curve shape matching, end-member selection and mixture modelling of AVIRIS and GER data for mapping surface mineralogy and vegetation communities. Airborne VISIBLE/Infrared imaging spectrometer (AVIRIS). In Airborne Geoscience Workshop Bibliographies, 20-21 May 1991 (Pasadena, CA: JPL Publications), pp. 158-162. 
MAselli, F., 1998, Multiclass spectral decomposition of remotely sensed scenes by selective pixel unmixing. IEEE Transactions on Geoscience and Remote Sensing, 36, pp. $1809-1820$.

NASH, J.C., 1990, The Choleski Decomposition. In Compact Numerical Methods for Computers: linear algebra and function minimization (Bristol: Adam Hilger), pp. 84-93.

Nordstrom, D.K., 1982, Aqueous pyrite oxidation and the consequent formation of secondary iron minerals. In Acid Sulfate Weathering, J.A. Kittrick, D.S. Fanning and L.R. Hossner (Eds) (Madison, Wisconsin: Soil Science Society of America), pp. $37-56$.

Okin, G.S., Siegel, H., Okin, W.J., Murray, B., Collier, J., Roberts, D.A., Miller, C.D., H., Painter, T.H. and Curkendall, D.W., 1999, The supercomputing visualization workbench for the analysis and classification of imaging spectrometer data. In AVIRIS Airborne Geoscience Workshop, 8-11 February 1999 (Pasadena, CA: JPL Publications), pp. 317-322.

Painter, T.H., Roberts, D.A., Green, R.O. and Dozier, J., 1998, The effect of grain size on spectral mixture analysis of show-covered area from AVIRIS data. Remote Sensing of Environment, 65, pp. 320-332.

Peddle, D.R., Forrest, G.H. and Ledrew, E.F., 1999, Spectral mixture analysis and geometric-optical reflectance modelling of boreal forest biophysical structure. Remote Sensing of Environment, 67, pp. 288-297.

Preissler, H., Bohbot, H., Mehl, W. and Sommer, S., 1998, MEDSPEC-A spectral database as a tool to support the use of imaging spectrometry data for environmental monitoring. In 1st EARSeL Workshop on Imaging Spectrometry, 6-8 October 1998, Zurich, Switzerland (Paris: EARSEL), pp. 455-462.

Press, W.H., Flannery, B.P., Teukolsky, S.A. and Vetterling, W.T., 1992, Cholesky Decomposition. In Numerical Recipes in FORTRAN: $\$ 2.9$ The art of scientific computing (Cambridge: Cambridge University Press), pp. 89-91.

Privette, J.L., Myneni, R.B. and Emery, M.J., 1996, Optimal sampling conditions for estimating grassland parameters via reflectance inversion models. IEEE Transactions on Geoscience and Remote Sensing, 34, pp. 272-284.

QIN, W. and GerstL, S.A.W., 2000, 3-D scene modelling of semidesert vegetation cover and its radiation regime. Remote Sensing of Environment, 71, pp. 197-206.

Richter, R., 1996, Atmospheric correction of DAIS hyperspectral image data. In Proceedings of SPIE, 2758, pp. 390-399.

Roberts, D.A., Smith, M.O., Adams, J.B. and Gillespie, A.R., 1991, Leaf spectral types, residuals, and canopy shade in an AVIRIS image. In 3rd Airborne Science Workshop, 20-21 May 1991 (Pasadena, CA: JPL Publications), pp. 43-50.

Roberts, D.A., Smith, M.O. and Adams, J.B., 1993, Green vegetation, nonphotosynthetic vegetation, and soils in AVIRIS data. Remote Sensing of Environment, 44, pp. $255-269$.

Roberts, D.A., Gardner, M., Church, R., Ustin, S., Scheer, G. and Green, R.O., 1998, Mapping chaparral in the Santa Monica Mountains using multiple endmember spectral mixture models. Remote Sensing of Environment, 65, pp. 267-279.

Sabol, D.E., Adams, J.B. and Smith, M.O., 1992, Quantitative subpixel spectral detection of targets in multispectral images. Journal of Geophysical Research, 25, pp. 2659-2672.

Schlaepfer, D., Schaepman, M.E. and Itten, K.I., 1998, Parge: parametric geocoding based on GCP-calibrated auxiliary data. Proceedings of SPIE, 3438, pp. 334-344.

Shimabukuro, Y.E., Batista, G.T., Mello, E.M.K., Moreira, J.C. and Duarte, V., 1998, Using shade fraction image segmentation to evaluate deforestation in Landsat Thematic Mapper images of the Amazon region. International Journal of Remote Sensing, 19, pp. 535-541. 
Smith, M.O., Susan, L.U., Adams, J.B. and Gillespie, A.R., 1990, Vegetation in deserts: I. A regional measure of abundance from multispectral images. Remote Sensing of Environment, 31, pp. 1-26.

Smith, M.O., Adams, J.B. and Sabol, D.E., 1994, Spectral mixture analysis: new strategies for the analysis of multispectral data. In Imaging Spectrometry - A tool for Environmental Observations. Euro Courses, Remote Sensing, J. Hill and J. Megier (Eds) (Boston, MA: Kluwer Academic), vol. 4, pp. 125-143.

Swayze, G.A., Smith, K.S., Clark, R.N., Sutley, S.J., Pearson, R.M., Vance, J.S., Hageman, P.L., Briggs, P.H., Meier, A.L., Singleton, M.J. and Roth, S., 2000, Using imaging spectroscopy to map acid mine waste. Environmental Science and Technology, 34, pp. 47-54.

Thomson, L.J. and Salisbury, J.W., 1993, The mid-infrared reflectance of mineral mixtures (7-14 mm). Remote Sensing of Environment, 17, pp. 37-53.

Ustin, S.L., Smith, M.O. and AdAms, J.B., 1993, Remote sensing of ecological processes: a strategy for developing and testing ecological models using spectral mixture analysis. In Scaling Physiological Processes: Leaf to Globe, J. Ehlringer and C. Field (Eds) (New York: Academic Press), pp. 339-357. 